\title{
Micellization and Interaction of Cationic Surfactants with Different Hydrophobic Group in Polar Organic Solvent
}

\author{
Osundiya Medinat Olubunmi ${ }^{1,}$ *, Olowu Rasaq Adewale ${ }^{1}$, Olaseni Segun Esan ${ }^{2}$, \\ Aboluwoye Christopher Olumuyiwa ${ }^{2}$ \\ ${ }^{1}$ Department of Chemistry, Lagos State University, Lagos, Nigeria \\ ${ }^{2}$ Department of Chemical Sciences, Adekunle Ajasin University, Akungba-Akoko, Nigeria
}

Email address:

bunmmyosundiya@yahoo.com (O. M. Olubunmi)

${ }^{*}$ Corresponding author

To cite this article:

Osundiya Medinat Olubunmi, Olowu Rasaq Adewale, Olaseni Segun Esan, Aboluwoye Christopher Olumuyiwa. Micellization and Interaction of Cationic Surfactants with Different Hydrophobic Group in Polar Organic Solvent. American Journal of Physical Chemistry. Vol. 9, No. 4, 2020, pp. 86-92. doi: 10.11648/j.ajpc.20200904.11

Received: September 7, 2020; Accepted: September 24, 2020; Published: October 14, 2020

\begin{abstract}
Surfactants are amphiphilic compounds that are widely used in various technological fields and biological systems. In many of these applications, mixtures of amphiphiles in organic solvents are employed for better performance The work aims at establishing the extent of ideality or otherwise in the mixed micelles of n-alkyl trimethylammonium bromide, (n $=14$ and 16 for TTABr and CTABr respectively), in water (AQ) and water-monoethanolamine (AQ-MEA) at different mole fractions $(0.1$ to 0.6$)$ and temperatures by Conductance measurement.: The values of the critical micelle concentration $(\mathrm{Cmx})$ obtained in AQ, and AQ-MEA were lower than that obtained assuming an ideal mixing system. Substantial deviation from ideality was observed in the presence of AQ-MEA at all temperatures, suggestive of more favourable association of the surfactants in MEA solvent. The composition $\left(\chi_{1}\right)$ of the mixed micelles obtained in the context of Rubingh's model showed that the micelles of CTABr were dominant, and the values of the interaction parameters $\left(\beta_{\mathrm{mx}}\right)$ were negative in AQ and AQMEA media with more synergistic interaction in the latter case at 0.1:0.9 mole fraction ratio at a particular temperature. The values of the activity coefficients $\left(\mathrm{f}_{\mathrm{TTABr}}, \mathrm{f}_{\mathrm{CTABr}}\right.$ ) were below unity, and the evaluation of the energetics of micellization showed that the process was spontaneous and feasible.
\end{abstract}

Keywords: Surfactant, Mixed-Micelles, Conductance Measurements

\section{Introduction}

The special interest in the study of surfactants originates from their technological and biological importance which revolve around the surface tension lowering (water) effect. $[1,2]$. A good number of different types of amphiphiles are mixed for the purpose of improved performance and costeffectiveness [2, 3]. The unique properties of cationic surfactants over other class of self-assembly [4] make them suitable in biological applications as solubilizing agents for organic compounds, and hydrophobic drugs, as disinfectants, and cleaning agents, also the positive charge on the surfaceactive segment of the molecule is also unaffected by the $\mathrm{pH}$ of the solution. [5-7].

As in the case of monomeric surfactants, the formation of micelles of two or more surfactants occur suddenly over a narrow range of concentration [1] due to changes in the physicochemical properties of the system [8]. The attractive hydrophobic interactions between the insoluble hydrocarbon tails give the driving force for aggregation, while the repulsive interaction among the hydrophilic head groups opposes aggregation [9]. A balance of these two major forces leads to micelle or mixed micelle formation as in the case of monomeric and mixed surfactants respectively [10-12].

The concentration of utmost importance in many formulations, and other technological applications is the critical micelle concentration (CMC), and is affected by structural and environmental factors such as hydrophobic, hydrophilic groups, temperature, electrolytes, nonelectrolytes and co-solvents $[13,14]$ therefore alteration of the solution properties by co-solvents have significant effect 
on the micellization process, and micelle formation [9]. Surfactant additives are essential in hands-on formulations in industries to regulate and improve the property of [15] products, nonetheless, the process of aggregation has been reported [16] to be less favourable in medium other than aqueous. Temperature and co-solvent were reported to have a distinct effect on the aggregation process [17], and hence the $\mathrm{CMC}$ values. The former play important roles on the level of hydration of both hydrophilic and hydrophobic group, while the effect of the later is largely based on whether the additives is a structure maker or breaker of the 3-dimensional arrangement of water molecules. [14, 17]. In the same manner, some polar organic co-solvent had been discovered to create a space between the polar end of the surfactant so as to minimize electrostatic repulsion, thereby prompt earlier micelle formation The surfactants and the co-solvent of choice (MEA) have enormous applications in chemical research [18]) and that attracted our interest in this work. Monoethanolamine (MEA), a polar organic compounds with functional groups that are hydrogen bonding inclined which according to Evans and coworker [19] a prerequisite for aggregation. The presence of the organic moiety in the structure is also of value as it can give surge to hydrophobic interactions. The structures of the surfactants and the cosolvent have been illustrated in Figure 1.
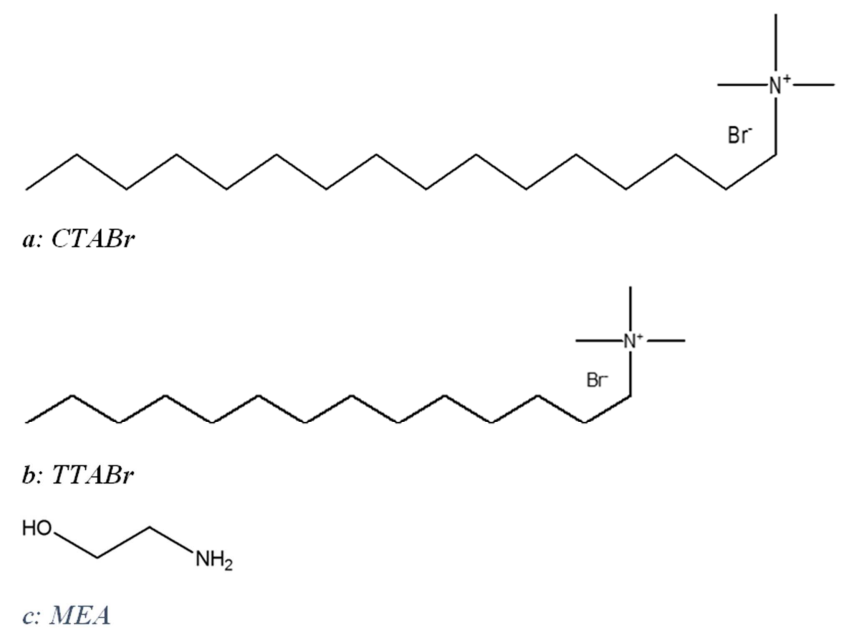

Figure 1. Cationic surfactants and MEA.

Although quite a number of reports are available in literature on binary mixtures of cationic surfactants in aqueous and aqueous-organic media $[13,14,17,20]$, but the fundamental investigations of the effect of monoethanolamine on the micellization of mixed cationic surfactants especially those of the linear quaternary ammonium halide origin has not been mentioned.

Electrical conductance method has been reported (21-23] to be the most accurate techniques and a better diagnostic tool [24] for the determination of CMC of ionic surfactants. This work is aimed at investigating the influence of co-solvent on the mixed micelles of cationic surfactants having the same hydrophilic but different tail groups, with a view to having a mixed micelle that will serve as a better adjuvant in herbicides, detergency formulation, and textile-dyeing. In this context, it is paramount to have a complete understanding of the interactions, and thermodynamic behaviour of these similarly structured surfactants. These were achieved by measuring the conductance of an increasing concentration of the solutions of surfactants mixture expressed in mole fraction ratios $\left(\alpha_{1}-\right.$ $\alpha_{6}$ ) in AQ, AQ-MEA media as a function of temperature.

\section{Experimental}

\subsection{Materials}

The cationic surfactants, $\mathrm{TTABr}$, and $\mathrm{CTABr}$ were purchased from Aldrich (USA) and Sigma chemicals. These surfactants are of the highest purity, that are commercially available, and they are therefore used without any further purification. Analytical grade MEA was also purchased from Sigma Aldrich and used as received. The water used in this work was re-distilled, with specific conductivity value that ranged between 1 and $4 \mu \mathrm{scm}^{-1}$ at room temperature. The composition of the solution was expressed in mole fraction $\left(\alpha_{i}\right)$, and the experiment was performed at temperatures between $298.1 \mathrm{~K}$ and $313.1 \mathrm{~K}$ at $5.1 \mathrm{~K}$ intervals. A Janway (model 4510) conductivity meter that was pre-calibrated with a standard solution $(0.01 \mathrm{~N})$ of $\mathrm{KCl}$ was used for the experiment.

\subsection{Methods}

Conductometric measurements were carried out using a dip-type probe of $1.0 \mathrm{~cm}^{-1}$ cell constant. $10 \mathrm{~cm}^{3}$ of the reference solution (water) or an appropriate amount of the co-solvent in water was taken in the conductance cell, and a $200 \mu l$ of a known concentration of TTABr-CTABr made with the reference solution was added, and allowed to equilibrate at a specific temperature before the onset of the experiment. The conductivity values were recorded when the solutions attained equilibrium. The conductivity of the increasing concentration of TTABr-CTABr in water (AQ), and water containing $0.2 \mathrm{v} / \mathrm{v} \%$ monoethanolamine $(0.2 \mathrm{v} / \mathrm{v} \%$ MEA) were measures at 298.1, 303.1, 308.1, and 313.1 K. The experimental error was $\pm 0.5 \%$. The above procedure was repeated for the entire mole fraction with freshly prepared stock solutions. All experiments were carried out under regulated temperature maintained $\pm 0.1^{\circ} \mathrm{C}$, and the $C_{m x}$ values of each mole fraction at a fixed temperature were determined from the interception point in the conductivity versus [TTABr-CTABr] curve

\section{Results and Discussion}

\subsection{Effect of Co-solvent on the Critical Micelle Concentration $\left(C_{m x}\right)$}

The electrical conductivity of different concentrations of TTABr-CTABr in $0.0 \mathrm{v} / \mathrm{v} \%$ and $0.2 \mathrm{v} / \mathrm{v} \%$ of MEA was measured to inquiring the effect of MEA on the micellization process and by extension the critical micelle concentration of 
the mixture at $298.1,303.1,308.1$, and $313.1 \mathrm{~K}$. The data obtained were plotted as a function of mixed surfactant concentration, to determine the inflection point, the $\mathrm{Cmx}$. The values obtained were summarized in Tables 1 and 2 respectively. The plots recorded two linear fragments, with different slopes, the pre mixed-micelle and post mixedmicellar regions which intercepted at the $\mathrm{Cmx}$. By subtracting the values obtained from the ratio of the slopes gave the extent of bromide-ion bound to the mixed micellar surface. However, the transition was not very spontaneous in MEA medium especially at $\alpha>0.3$ and temperatures above 298.1 K. This observation which made the conventional plot inadequate was similar to those reported in our previous work [25], and was treated accordingly using the Carpena method to obtain the exact point of inflection, which corresponds to the $\mathrm{Cmx}[26]$.

The values of the experimental critical mixed micelle concentration ( $\mathrm{Cmx}$ ) for TTABr-CTABr in aqueous medium were slightly lower than the values obtained from ideal mixing. The non-ideality in the mixed-micelle was due to molecular interactions between the surfactant monomers. The decrease in the values of $\mathrm{Cmx}$ as compared with the ideal solution was quite pronounced upon the addition of $0.2 \mathrm{v} / \mathrm{v} \%$ MEA. Hence, departure from ideality was more apparent in AQ-MEA media. In addition to monomeric interactions in AQ medium, the substantial decrease in the $\mathrm{Cmx}$ values when co-solvent was involved could be ascribed to (i) reinforcement of the 3-D structure of water by MEA, and its ability to intercalate [25] the mixed-micellar surface by increasing through replacement of some water molecules [27] the distance between the polar head group, and consequently reduced the electrostatic repulsion. (ii) In the same fashion the alkyl portion in MEA enhanced the hydrophobicity of the system through van der Waals interactions [28] among the hydrophobic/solvophobic groups thus promoting aggregation with a noticeable decrease in the values of $\mathrm{C}_{\mathrm{mx}}$ Similar trend upon the addition of some organic solvents to Gemini surfactant was reported in literature [29]

Table 1. The solution mole fraction $(\alpha)$, critical micelle concentration (Cmx) micellar composition $\left(\chi_{I}\right)$, interaction parameter $\left(\beta_{m x}\right)$, and activity coefficients $\left(f_{\text {TTABr }}, f_{\text {CTABr }}\right)$ of TTABr-CTABr in $0.0 \mathrm{v} / \mathrm{v} \% \mathrm{MEA}$.

\begin{tabular}{llllll}
\hline $\boldsymbol{\alpha}$ & $\mathbf{C m x}(\mathbf{m M})$ & $\boldsymbol{\chi}_{\mathbf{1}}$ & $\boldsymbol{- \beta}_{\mathbf{m x}}$ & $\mathbf{f}_{\mathbf{T T A B r}}$ & $\mathbf{f}_{\mathbf{C T A B r}}$ \\
\hline & $298.1 \mathrm{~K}$ & & & & \\
0.1 & 0.868 & 0.111 & 1.925 & 0.219 & 0.977 \\
0.2 & 0.943 & 0.139 & 1.313 & 0.379 & 0.975 \\
0.3 & 0.995 & 0.190 & 1.253 & 0.439 & 0.956 \\
0.4 & 1.016 & 0.255 & 1.456 & 0.446 & 0.909 \\
0.5 & 1.113 & 0.296 & 1.262 & 0.536 & 0.895 \\
0.6 & 1.179 & 0.363 & 1.487 & 0.547 & 0.822 \\
& $303.1 \mathrm{~K}$ & & & & \\
0.1 & 0.894 & 0.093 & 1.597 & 0.269 & 0.987 \\
0.2 & 0.959 & 0.130 & 1.169 & 0.413 & 0.980 \\
0.3 & 1.010 & 0.183 & 1.152 & 0.464 & 0.962 \\
0.4 & 1.062 & 0.240 & 1.217 & 0.496 & 0.932 \\
0.5 & 1.149 & 0.293 & 1.198 & 0.549 & 0.902 \\
0.6 & 1.259 & 0.351 & 1.199 & 0.606 & 0.863 \\
& $308.1 \mathrm{~K}$ & & & & \\
\hline
\end{tabular}

\begin{tabular}{llllll}
\hline $\boldsymbol{\alpha}$ & $\mathbf{C m x}(\mathbf{m M})$ & $\boldsymbol{\chi}_{\mathbf{1}}$ & $\boldsymbol{- \beta}_{\mathbf{m x}}$ & $\mathbf{f}_{\text {TTABr }}$ & $\mathbf{f}_{\mathbf{C T A B r}}$ \\
\hline 0.1 & 0.918 & 0.076 & 1.263 & 0.339 & 0.993 \\
0.2 & 1.018 & 0.090 & 0.533 & 0.633 & 0.996 \\
0.3 & 1.058 & 0.159 & 0.826 & 0.558 & 0.979 \\
0.4 & 1.082 & 0.233 & 1.113 & 0.519 & 0.941 \\
0.5 & 1.206 & 0.278 & 0.959 & 0.607 & 0.929 \\
0.6 & 1.284 & 0.347 & 1.121 & 0.622 & 0.875 \\
& $313.1 \mathrm{~K}$ & & & & \\
0.1 & 0.946 & 0.052 & 0.754 & 0.508 & 0.946 \\
0.2 & 1.029 & 0.083 & 0.434 & 0.695 & 0.997 \\
0.3 & 1.072 & 0.152 & 0.727 & 0.593 & 0.983 \\
0.4 & 1.109 & 0.224 & 0.977 & 0.555 & 0.952 \\
0.5 & 1.213 & 0.276 & 0.928 & 0.615 & 0.932 \\
0.6 & 1.308 & 0.343 & 1.034 & 0.640 & 0.885 \\
\hline
\end{tabular}

Table 2. The solution mole fraction ( $\alpha)$, critical micelle concentration (Cmx) micellar composition $\left(\chi_{1}\right)$, interaction parameter $\left(\beta_{m x}\right)$, and activity coefficients $\left(f_{\text {TTABr }}, f_{C T A B r}\right)$ of TTABr-CTABr in $0.2 v / v \%$ MEA.

\begin{tabular}{|c|c|c|c|c|c|}
\hline$\alpha$ & $\operatorname{Cmx}(\mathrm{mM})$ & $\chi_{1}$ & $-\boldsymbol{\beta}_{\mathrm{mx}}$ & $\mathbf{f}_{\mathrm{TTABr}}$ & $\mathbf{f}_{\mathrm{CTABr}}$ \\
\hline & $298.1 \mathrm{~K}$ & & & & \\
\hline 0.1 & 0.598 & 0.234 & 4.501 & 0.072 & 0.781 \\
\hline 0.2 & 0.626 & 0.268 & 3.804 & 0.131 & 0.760 \\
\hline 0.3 & 0.762 & 0.273 & 2.739 & 0.235 & 0.816 \\
\hline 0.4 & 0.707 & 0.331 & 3.196 & 0.293 & 0.705 \\
\hline 0.5 & 0.705 & 0.371 & 3.281 & 0.273 & 0.637 \\
\hline 0.6 & $\begin{array}{l}- \\
303.1 \mathrm{~K}\end{array}$ & - & - & - & - \\
\hline 0.1 & 0.546 & 0.251 & 4.995 & 0.061 & 0.729 \\
\hline 0.2 & 0.571 & 0.284 & 4.261 & 0.113 & 0.709 \\
\hline 0.3 & 0.682 & 0.295 & 3.289 & 0.195 & 0.752 \\
\hline 0.4 & 0.643 & 0.343 & 3.623 & 0.209 & 0.652 \\
\hline 0.5 & 0.639 & 0.368 & 3.168 & 0.282 & 0.651 \\
\hline 0.6 & $\begin{array}{l}- \\
308.1 \mathrm{~K}\end{array}$ & - & - & - & - \\
\hline 0.1 & 0.512 & 0.262 & 5.332 & 0.055 & 0.694 \\
\hline 0.2 & 0.553 & 0.289 & 4.422 & 0.107 & 0.691 \\
\hline 0.3 & 0.618 & 0.310 & 3.757 & 0.167 & 0.697 \\
\hline 0.4 & 0.557 & 0.359 & 4.247 & 0.174 & 0.579 \\
\hline 0.5 & 0.526 & 0.384 & 3.911 & 0.227 & 0.561 \\
\hline 0.6 & $\begin{array}{l}- \\
313.1 \mathrm{~K}\end{array}$ & - & - & - & - \\
\hline 0.1 & 0.455 & 0.279 & 5.927 & 0.046 & 0.611 \\
\hline 0.2 & 0.462 & 0.321 & 5.643 & 0.074 & 0.558 \\
\hline 3 & 0.558 & 0.324 & 4.235 & 0.145 & 0.641 \\
\hline 0.4 & 0.679 & 0.367 & 3.377 & 0.226 & 0.682 \\
\hline 0.5 & 0.744 & 0.337 & 3.149 & 0.284 & 0.654 \\
\hline 0.6 & - & - & - & - & - \\
\hline
\end{tabular}

However, an increase in the values of $\mathrm{Cmx}$ which was linear with an increase in the solution mole fraction ( $\alpha$ i) was noticed in both media as a result of an increase in the bulk concentration of the mixed surfactants in the solution. This resulted into more electrostatic repulsion by virtue of an increase in the surface charge density at the mixed micellar interface (corolla). Furthermore, an increase in $\alpha \mathrm{i}$ also resulted into a decrease in the concentration of the more surface active constituents (CTABr) which has a higher inclination to micelle formation due to its longer hydrocarbon chain [29-30]. These effects were however, beclouded in AQMEA system as a result of the solvent characteristics, and the 
extent to which they were non-ideal could be found in Figure 1 which illustrated the comparison at $298.1 \mathrm{~K}$.

An increase in the $\mathrm{Cmx}$ values was also the trend as the temperature of the system was increasing (Tables 1 and 2). De-solvated hydrophilic group favours aggregation and a decrease in hydration through the disruption of the structure of water in the vicinity of the hydrophobic parts opposes micellization. The predominance of these factors determines the trend of the critical micelle concentration [15] In this study, the results showed that the second factor was responsible for the values obtained in $\mathrm{AQ}$, as a shift in equilibrium (monomer $\rightleftharpoons$ mix-micelle) in favour of the reactant [31] at elevated temperature was noticed (Table 1), while the first factor came to play in AQ-MEA as the temperature was increasing (Table 2) owing to solvophobic effect [32].

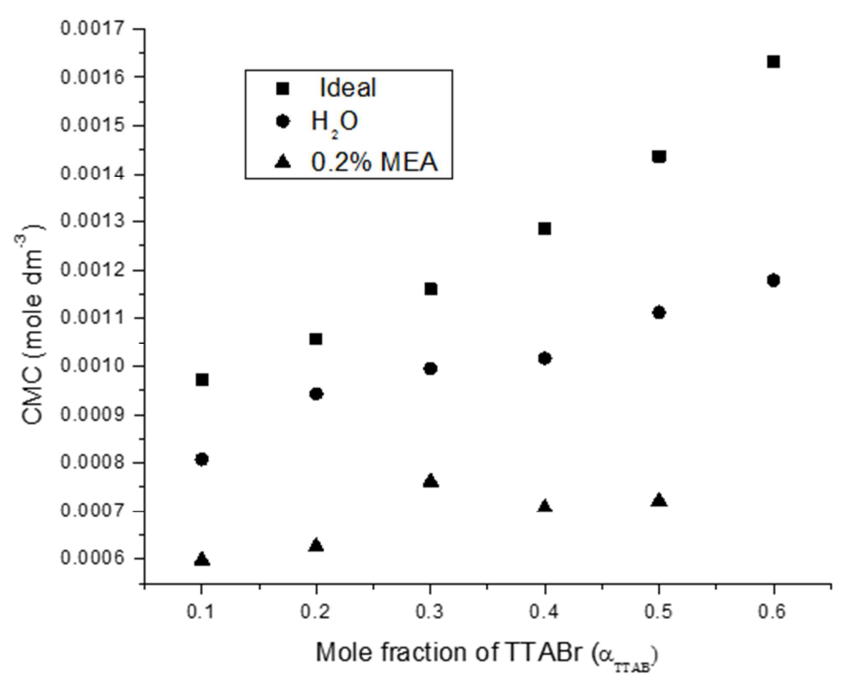

Figure 2. The plot of Cmx of TTABr-CTABr in AQ, AQ-MEA as a function of mole fraction of at $298.1 \mathrm{~K}$.

The theoretical critical micelle concentration of the ideal mixture of TTABr and $\mathrm{CTABr}\left(C M C^{i d}\right)$ can be calculated with the aid of Clint's equation [33)

$$
\frac{1}{C M C^{i d}}=\sum_{i=1}^{N=2} \frac{\alpha_{i}}{C M C_{i}}
$$

Where $\alpha_{i}$ is the bulk mole fraction of the ith monomeric components, $\mathrm{CMC}_{\mathrm{i}}$, its critical micelle concentrations of the pure forms (TTABr and $\mathrm{CTABr}$ ) and $\mathrm{CMC}^{\mathrm{id}}$, the critical micelle concentration for the ideal mixture.

However, in view of the departure from ideal system ((Figure 1), Clint's equation was modified by Rubingh [34] with an inclusion of the activity coefficient $\left(f_{i}\right)$ to Eq. 1 in order to account for the nature and the degree of interactions which led to non-ideality in the micelle mixture, and was calculated using these equations,

$$
\begin{gathered}
f_{T T A B r}=\exp \left[\beta_{m x}\left(1-\chi_{1}\right)\right]^{2} \\
f_{C T A B r}=\exp \left(\beta_{m x} \chi_{1}{ }^{2}\right)
\end{gathered}
$$

Where $f_{\text {TTABr }}, f_{C T A B r}$ are the activity coefficients for the first component (TTABr) and second component (CTABr) in the mixed micelle.

Equation 3 was iteratively solved to obtain the values of the micellar mole fraction $\left(\chi_{1}\right)$ of component one (TTABr) in the mixture, which in turn was used to evaluate the molecular interaction parameters $\left(\beta_{m x}\right)$. The values of $\beta_{m x}$ (dimensionless) give information as regards the direction and the magnitude of association between the two surfactants that forms the mixed-micelle. A negative $\left(\beta_{\mathrm{mx}}<0\right)$ and positive $\left(\beta_{\mathrm{mx}}>0\right)$ values of beta indicate attractive or repulsive interactions between the molecules of the surfactants respectively, while an interaction parameter of zero $(\beta \mathrm{mx}=0)$ is an indication of no reaction possibly due to hydrophobic/ hydrophilic mismatch [35]

$$
\begin{gathered}
\frac{\chi_{1}^{2} \ln \left(\alpha_{1} C_{m x} / C M C_{1} \chi_{1}\right)}{\left(1-\chi_{1}\right)^{2} \ln \left[C_{m x}\left(1-\alpha_{1}\right) / C M C_{2}\left(1-\chi_{1}\right)\right]}=1 \\
\beta_{m x}=\frac{\ln \left[\left(\alpha_{1} c_{m x}\right) /\left(c m c_{1} \chi_{1}\right)\right]}{\left(1-\chi_{1}\right)^{2}}
\end{gathered}
$$

$\mathrm{CMC}_{1}, \mathrm{CMC}_{2}$, are the theoretical critical micelle concentration for $\mathrm{TTABr}$ and $\mathrm{CTABr}$ respectively.

\subsection{Composition $\left(\chi_{1}\right)$, Interaction Parameters $\left(\beta_{m x}\right)$, and Activity Coefficients $\left(f_{\text {TTABr, }} f_{\text {CTABr }}\right)$ of Mixed-micelles}

Analyses of mixed micellar composition has been reported in aqueous medium to be dominated by the micelle of the surfactant with longer chain length [36-37]. In this work, the composition of the mixed micelle obtained upon further characterization due to non-ideality revealed that the micelle of CTABr was dominant in AQ and AQ-MEA media, and the values were both composition and temperature dependent (Tables 1-2). This behaviour was extensively discussed in literature $[35,38,39]$. Nonetheless, in contrast to the trend in AQ medium the values of $\chi_{1}$ were found to increase with an increase in temperature in MEA. It was obvious from the results that the mixed micelles contained larger fraction of the more surface active, and longer chain length surfactant (CTABr) over the whole range of compositions studied in both media. Literature showed some semblance to these findings $[11,36]$.

The results of the non-ideal behaviour associated with cooperative molecular interaction (synergism) was obtained from equation 4 and also included in Tables 1 and 2 for AQ and AQ-MEA respectively. The negative values obtained was an indication of attractive interactions between the two cationic surfactants that formed the mixed-micelles, with the more surface active (CTABr) being the first to aggregate. The stepwise insertion of the micelles of TTABr into the existing micelles of CTABr was discovered to be accompanied by favourable attractive forces This synergistic interactions, an indication of a more compact cluster aggregates [40]. The values of the $\beta_{m x}$ were negative at any given temperature, but retardation in the mixed micelle formation was also collaborated with a decrease in the interaction parameter $\left(\beta_{\mathrm{mx}}\right)$. as synergism became smaller in magnitude with an increase in temperature and composition $\left(\alpha_{\mathrm{TTABr}}\right)$ in aqueous 
medium. This may be due to the crowdedness of the polar head group at the aggregated surface. Nonetheless, this effect was restrained upon the addition of MEA as the values of $\beta_{\mathrm{mx}}$.

became increasingly more negative (Table 2). This increased synergism could be linked to the ability of MEA to act as a spacer thereby stabilizing the mixed-micelles by reducing the electrostatic repulsion at the surface, which was achieved [14] through electro-neutralization of the polar head groups at the stern layer by the counter-ion $\left(\mathrm{Br}^{-}\right.$.) However, an overall maximum cooperative (synergism) association was found when the mole ratio of $\mathrm{TTABr}$ to $\mathrm{CTABr}$ was at 0.1:0.9.

All the values of activity coefficient were less than unity, with $\mathrm{f}_{\mathrm{TTABr}}<<\mathrm{f}_{\mathrm{CTABr}}$, an indication that further collaborated non-ideality.

\subsection{Thermodynamics of Mixed-Micelles}

The feasibility of the micellization process with and without the organic co-solvent was examined by determining the relevant thermodynamic quantities using the phase separation model [34] In accordance with the regular solution theory, the relationship between the excess free energy $\left(G^{E}\right)$ the excess enthalpy $\left(H^{E}\right)$ and the changes in Gibb's free energy and enthalpy of micellization are given by

$$
\begin{gathered}
\left.G^{E}=H^{E}=\Delta H_{m x}=R T \chi_{1} \ln f_{T T A B r}+\left(1-\chi_{1}\right) \ln f_{C T A B r}\right) \\
\Delta G_{M x}=R T\left(\chi_{1} \ln \left(\chi_{1} f_{T T A B r}\right)+\left(1-\chi_{1}\right) \ln \left(1-\chi_{1}\right) f_{C T A B r}\right.
\end{gathered}
$$

The entropy change for the micellization process was obtained from the relation;

$$
\Delta S_{m x}=\frac{1}{T}\left(\Delta H_{m x}-\Delta G_{m x}\right)
$$

The results of energetics of reaction showed that the micellization process was thermodynamically feasible (Tables 3 and 4).

\begin{tabular}{|c|c|c|c|c|}
\hline$\alpha$ & $\begin{array}{l}\mathrm{Cmx} \\
(\mathrm{mM})\end{array}$ & $\frac{-\Delta \boldsymbol{G}_{\boldsymbol{m x}}}{\boldsymbol{K J m o l}^{-1}}$ & $\frac{-\Delta H_{m x}}{\text { KJmol }^{-1}}$ & $\frac{\Delta S_{m} x}{\operatorname{Jmol}^{-1} K^{-1}}$ \\
\hline & $298.1 \mathrm{~K}$ & & & \\
\hline 0.1 & 0.868 & 1.333 & 0.469 & 2.898 \\
\hline 0.2 & 0.943 & 1.387 & 0.388 & 3.352 \\
\hline 0.3 & 0.995 & 1.683 & 0.478 & 4.042 \\
\hline 0.4 & 1.016 & 2.094 & 0.686 & 4.720 \\
\hline 0.5 & 1.113 & 2.157 & 0.651 & 5.050 \\
\hline 0.6 & $\begin{array}{l}1.179 \\
303.1 \mathrm{~K}\end{array}$ & 2.476 & 0.852 & 5.447 \\
\hline 0.1 & 0.894 & 1.110 & 0.331 & 2.573 \\
\hline 0.2 & 0.959 & 1.308 & 0.334 & 3.212 \\
\hline 0.3 & 1.010 & 1.633 & 0.433 & 3.957 \\
\hline 0.4 & 1.062 & 1.048 & 0.559 & 4.482 \\
\hline 0.5 & 1.149 & 2.151 & 0.627 & 5.028 \\
\hline 0.6 & $\begin{array}{l}1.259 \\
308.1 \mathrm{~K}\end{array}$ & 2.321 & 0.688 & 5.388 \\
\hline 0.1 & 0.918 & 0.916 & 0.227 & 2.236 \\
\hline 0.2 & 1.018 & 0.889 & 0.115 & 2.516 \\
\hline 0.3 & 1.058 & 1.405 & 0.283 & 3.642 \\
\hline 0.4 & 1.082 & 1.902 & 0.511 & 4.513 \\
\hline 0.5 & 1.206 & 2.031 & 0.492 & 4.914 \\
\hline
\end{tabular}

Table 3. Thermodynamic Behaviour of Micellization of TTABr-CTABr in 0.0 $v / v \% M E A$.

\begin{tabular}{lllll}
\hline $\boldsymbol{\alpha}$ & $\begin{array}{l}\mathbf{C m x} \\
(\mathbf{m M})\end{array}$ & $\frac{-\Delta \boldsymbol{G}_{\boldsymbol{m} \boldsymbol{x}}}{\mathbf{K J m o l}^{\mathbf{1}}}$ & $\frac{-\Delta \boldsymbol{H}_{\boldsymbol{m} \boldsymbol{x}}}{\mathbf{K J m o l}^{-\mathbf{1}}}$ & $\frac{\Delta \boldsymbol{S}_{\boldsymbol{m}} \boldsymbol{x}}{\mathbf{J m o l}^{-\mathbf{1}} \boldsymbol{K}^{\mathbf{- 1}}}$ \\
\hline 0.6 & 1.284 & 2.299 & 0.645 & 5.346 \\
& $313.1 \mathrm{~K}$ & & & \\
0.1 & 0.946 & 0.629 & 0.096 & 1.699 \\
0.2 & 1.029 & 0.830 & 0.086 & 2.378 \\
0.3 & 1.072 & 1.354 & 0.245 & 3.543 \\
0.4 & 1.109 & 1.827 & 0.443 & 4.442 \\
0.5 & 1.213 & 2.016 & 0.482 & 4.898 \\
0.6 & 1.308 & 2.281 & 0.607 & 5.346 \\
\hline
\end{tabular}

\begin{tabular}{|c|c|c|c|c|}
\hline$\alpha$ & $\begin{array}{l}\mathrm{Cmx} \\
(\mathrm{mM})\end{array}$ & $\frac{-\Delta \boldsymbol{G}_{\boldsymbol{m x}}}{\boldsymbol{K J \boldsymbol { J o l }}^{-1}}$ & $\frac{-\Delta H_{m} x}{K^{\prime} m l^{-1}}$ & $\frac{\Delta S_{m} x}{\operatorname{Jmol}^{-1} K^{-1}}$ \\
\hline & $298.1 \mathrm{~K}$ & & & \\
\hline 0.1 & 0.598 & 3.344 & 1.995 & 4.523 \\
\hline 0.2 & 0.626 & 3.289 & 1.848 & 4833 \\
\hline 0.3 & 0.762 & 2.799 & 1.346 & 4.874 \\
\hline 0.4 & 0.707 & 3.327 & 1.754 & 5.279 \\
\hline 0.5 & 0.705 & 3.536 & 1.902 & 5.486 \\
\hline 0.6 & - & - & - & - \\
\hline & $303.1 \mathrm{~K}$ & & & \\
\hline 0.1 & 0.546 & 3.785 & 2.366 & 4.684 \\
\hline 0.2 & 0.571 & 3.685 & 2.181 & 4.961 \\
\hline 0.3 & 0.682 & 3.250 & 1.722 & 5.043 \\
\hline 0.4 & 0.643 & 3.682 & 2.061 & 5.346 \\
\hline 0.5 & 0.639 & 3.515 & 1.857 & 5.469 \\
\hline 0.6 & $-\overline{308.1 \mathrm{~K}}$ & - & - & - \\
\hline 0.1 & 0.512 & 4.110 & 2.637 & 4.782 \\
\hline 0.2 & 0.553 & 3.868 & 2.328 & 4.999 \\
\hline 0.3 & 0.618 & 3.645 & 2.059 & 5.147 \\
\hline 0.4 & 0.557 & 4.178 & 2.505 & 5.428 \\
\hline 0.5 & 0.526 & 4.077 & 2.371 & 5.537 \\
\hline 0.6 & $-313.1 \mathrm{~K}$ & - & - & - \\
\hline 0.1 & 0.455 & 4.642 & 3.100 & 4.922 \\
\hline 0.2 & 0.462 & 4.841 & 3.207 & 5.218 \\
\hline 0.3 & 0.558 & 4.051 & 2.411 & 5.237 \\
\hline 0.4 & 0.679 & 3.629 & 1.965 & 5.313 \\
\hline 0.5 & 0.744 & 3.617 & 1.904 & 5.469 \\
\hline 0.6 & - & - & - & - \\
\hline
\end{tabular}

Table 4. Thermodynamic Behaviour of Micellization of TTABr-CTABr in 0.2 $v / v \% M E A$.

All the values of the thermodynamics parameters showed that the process of micellization was spontaneous and thermodynamically feasible in AQ, and AQ-MEA (Tables 3, 4 ), with aggregation being more spontaneous in the later at elevated temperature. The values of $\Delta G_{m x}$ and $\Delta S_{m x}$ were all negative and positive respectively. The increased randomness displayed in AQ-MEA was a clear indication of increased hydrophobic interactions between the quaternary amine cationic surfactants and the continuous liberation of water molecule during the process of concealment of the hydrocarbon tail both from the surfactants and the organic solvent into the interior of the mixed-micelles, hence the process became increasingly entropy driven. In AQ-MEA the exothermicity of the process was at the optimum when the temperature was raised, due to continuous bond broken and bond formation of the three dimensional structure of water.

\section{Conclusion}

The surfactants mixture investigated have the same head group, hence, results obtained clearly displayed non-ideality 
due to tail-tail interactions, and contribution from the cosolvent. There were more synergistic interactions in the mixed state when the reaction medium was reinforced with MEA, a situation that led to further departure from ideal state. MEA contributed positively to the micellization process of TTABr-CTABr as the organic group in the structure augmented hydrophobic interaction especially at higher temperature. Nevertheless, our findings showed that TTABr-CTABr MEA mixed micelles did not cooperate beyond 0.5 mole fraction, and an attempt to increase its percentage led to non aggregation. The mixed micelle is cost effective especially at 0.1: 0.9 TTABr-CTABr. With the aid of appropriate models, the properties of mixed cationic surfactants could be improved with the use of MEA.

The authours gratefully acknowledged the support received from the academic and technical staff of the Department of Chemical Sciences, Adekunle Ajasin University Akungba.

\section{References}

[1] Gholamreza, D. N., Mohammadreza, H., and Bibi S. F. B. (2007). The Effect of Temperature on Thermodynamic Parameters of micellization of Some surfactants. Journal of Applied Sciences, 7: (1) 47-52.

[2] Amit, K, T. S., and Sonu, S. K. S. (2013). Aggregation behavior and thermodynamics of mixed micellization of gerini surfactants with a room temperature ionic liquid in water and water-organic solvent mixed media. J. Chem. Thermodynamics 60, 29-40.

[3] Dharmesh, V., Vijay, P., Anita, B., Pratab, B., and Martun, S. V. (2004). Mixed micelles of cationic surfactants and sodium cholate in water. Indian. Journal of Biochemistry \& Biophysics 41, 107-112

[4] Jitendra, M., Dharmes, V., and Prashant, B. (2005). Aggregation behavior of quaternary salt based cationic surfactants. Thermochimica Acta. 428 (1-2): 147-155.

[5] Milton, J. R. (2004). Interfacial phenomenon second edition, Wiley Publisher, 12-25.

[6] Badache, L., Lehanine, Z., Abderrahmane, W. N. (2012). Synthesis and surface properties study of a series of cationic surfactants with different hydrophobic chain length. Journal of surfactant and detergent. 15, 715-720.

[7] Owoyomi, O., Ige, J., and Soriyan, O. O. (2011). Thermodynamics of Micellization of nalkyltriphenylphosphonium Bromide: A Conductometric Study. Chemical Science Journal, CSJ-25, 1-13

[8] Lei, H., and Samasundaran (1997). Theoretical Model and Phase Behavior for Binary Surfactant Mixture. Langmuir 13 (25): 6683-6688

[9] Aswal, V. K., and Goyal, P. S. (2002). Role of counterion distribution on the structure of micelles in aqueous salt solutions: Small angle neutron scattering study. Chemical Physics letters. 357, 491-497

[10] Josh, J. V., Aswal, V. K., Bahadur, P., and Goyal, P. S. [2012]. Role of counterion of surfactant molecule on the micellar structure in aqueous solution. Current Science. 83: (1) 47-49

[11] Ortonia, O., D’Errico, G., Vitagliano, V., and Costantino, L. (2002). Mixed micellar aggregates of nonionic and anionic surfactants with short hydrophobic tails: A microcalorimetric study. Journal of colloid and interface. 249 (2): 481-488.

[12] Osundiya, M. O., and Olaseni, S. E. (2016). Aggregational attitude of Hexadecyltrimethylammonium Bromide in Aqueous Solution of Sodium salt at $298.1 \mathrm{~K}$. International journal of Chemistry and Materials Research 4 (4): 27-34.

[13] Nighat, N., Mohammed, S., and Arif, K. (2009). Micellization of Cationic Surfactant cetyltrimethylammoniumBeomide in Mixed Water-Alcohol. Journal of Dispersion Science and Technology. 51-55.

[14] Ashish, S., and Kallol, K. G. (2008). Micellization of cethyltriphenylphosphonium bromide surfactant in binary aqueous solvents. Journal of surfactant and detergent. 11, 287-292

[15] Chauhan, S., Kaur, M., Kumor, K., and Chauhan, M. S. (2014). Study of the effect of electrolyte and temperatureon the critical micelle concentration of dodecyltrimethylammonium bromide in aqueous medium. Journal of chemical thermodynamics, 178, 175-181

[16] Ali, A., Nabi, F., Maliki, N. A., Tesneem, S., and Uzair, S. (2014). Study of Micellization of Sodiumdodecylsulfatein non-aqueous media containing lauric acid and dimethyl sulfoxide. Journal of surfactant detergent. 17, 151-160

[17] Olaseni, S. E., Oladoja, N. A., Ololade I. A., Aboluwoye, C. O., and Osundiya, M. O. (2012). Micellization of Cetyltrimethylammonium bromide in aqueous-organic media. Chemical science journal, 52, 1-11.

[18] Mishra, B. K., Mukherjee P., Dash, S., Patel S., and Pati, H. N. (2009). Alkylation of ethanolamine: An approach to a novel class of functional surfactant synthesis communication 39, 2529-2539.

[19] Evans, D. F., Wennestrom, H. (1994). 'The Colloidal Domain where Physics, Chemistry and Biology Meets, 'VCH, New York

[20] Misra, P. K., Mishra, B. K., and Bebera, G. B. (1991). Micellization of ionic surfactants in tetrahydrofuranwater and acetonitrile-water mixed solvent systems. Colloid and surfaces. 57 (1): 1-10.

[21] Owoyomi, O., Alao, O., Soriyan, O., and Ogunlusi G. (2013). Micellization thermodynamics of sodium lauroylsarcosinate in water-alcohol binary mixtures. Physics and Chemistry of liquids. 1-8.

[22] Gargi, B. Y., Soumen, G., and Satya., P. M. (2010). Ternary mixtures of alkyltriphenylphosphonium bromide $\left(\mathrm{C}_{12} \mathrm{TPB}\right.$. $\mathrm{C}_{14} \mathrm{TPB}, \mathrm{C}_{16} \mathrm{TPB}$ ) in aqueous medium, their interfacial, bulk and fluorescence quenching behaviour. Journal Chem. Sci. 122 (2), 109-117.

[23] Ana, K., Bojan, S., and Mariji, B., R. (2012). What Affect the Degree of Micelle Ionization: Conductivity Study of Alkyltrimrthylammonium Chloride. Acta. Slov. 59, 564-570.

[24] Prasad, M., Moulik, S. P., and Palepu, R. (2005). Selfaggregation of binary mixtures of alkyltriphenylphosphonium bromide: a critical assessment in favor of more than one kind of micelle formation. Journal of colloid and interface science, $284, .658-666$ 
[25] Olaseni, S. E., Osundiya, M. O., Aboluwoye, C. O., Owoyomi, O. (2015) Effect of Temperarure and Tetramethyammonium Bromide Salt on the Micellization of Cetyltrimethylammonium Bromide in Aqueous Medium: A conductometric Studies. International Journal of Thermodynamics. 18 (4): 246-252

[26] Carpena, P. Aguiar, J., Bemaola-Garvan, P. and Ruiz Langmuir, C. (2002) 18, 60546.

[27] Carnero, C. R., and Aguiar, J. (2001) Interaction, Stability, and Microenvironment Properties of Mixed Micelles of Triton X100 and n-Alkyltrimethylammonium Bromide. Influence of Alkyl Chain Length. Langmuir. 16, 7946-7953

[28] Li, F., Gan-Zuo Li., and Jian-Bo, C. (1998). Synergism in mixed zwitterionic-anionic surfactant solutions and the aggregation numbers of the mixed micelles. Colloids and surface A. Physicochemical and Engineering Aspects. 145, 167-174.

[29] Amit, K., Tiwari, S., and Subit, K. S. (2013) Aggregation behavior and thermodynamics of mixed micellization of gemini surfactants with room temperature ionic liquid in water and water-organic solvent media. Journal of Chemical Thermodynamics. 60, 29-40

[30] Soumen, G., Anupam, D., B., Gobinda, C. D., ansAkhil, R. D. (2011) The journal of Physical Chemistry B. 115, 1109811112 .

[31] Sansamwal, P. K. (2006). Effect of co-solutes physicoChemical properties of surfactant solutions. Journal of Science industrial research 57-64

[32] Bakshi. M. S., and Kur, G; (2000). Mixed micelles of hexadecylpyridinium bromide in aqueous glycol oligomers. Journal of surfactants and Detergent, 3 (2): 162-166.

[33] Clint, J. H. (1990). 'Mixed micelle theory as an aid to surfactant formulation' in the structure, Dynamics and Equilibrium properties of Colloidal Systems, D. M. Bloor, Ed, Kluwer Academic, London. 7184.

[34] Holland, H. D., and Rubingh, D. (1992). 'Chapter 1, An overview' in Mixed Surfactant system, H. D. Holland and D. Rubingh, Eds ACS Symposium Series An overview. American chemical society symposium series. Washington D. C. 501: 2-30.

[35] Ray, G. B., Chakraborty, I., Ghosh, S., and Moulik, S. K. (2007). A critical and comprehensive assessment of interfacial and bulk properties of aqueous binary mixtures of anionic surfactants, sodiumdodecyl sulfate, and sodiumdodecylbenzene sulfonate. Colloid and polymer science. $285,457-469$.

[36] Merta, J. and Stenius, P. (1999). Interactions between cationic starch and mixed anionic surfactants. Colloid and surfaces a: physico-chemical and engineering Aspect, 149, 367-377.

[37] Maria, M., Amalia, R., Maria-Del, M. G., and Maria, L. M. (2004). Conductometric, surface tension, and kinetic studies in mixed SDS-Tween 20 and SDS-SB3-12 micellar solutions. Langmuir 20, (25): 10858-10867.

[38] Rodena, E., Valiente, and Villafruella, M. Dd. s. (1999). Different theoretical approaches for the study of the mixed tetra ethylene glycol mono-n-dodecyl ether/hexadecyltrimethylammonium bromide micelles. $J$. Phys. Chem. B, 103, 4549-4554.

[39] Al-Wardian, A., Glenn, K. M., and Palepu, R. M. (2004). Thermodynamic and interfacial properties of binary cationic mixed systems. Colloid and surface A: Physicochemical. Eng. Aspect, 247, 115-123.

[40] Roman-Guerrero, A., Vernon-Carter, E. J., and Demarse, N. A. (2010). Thermodynamics of micelle formation. TA Instruments-Waters LLC, 5, 1-6. 